\title{
EL TRABAJO COLABORATIVO EN LA EDUCACIÓN SUPERIOR: UNA COMPETENCIA PROFESIONAL PARA LOS FUTUROS DOCENTES*
}

\author{
Javier Fombona Cadavieco ${ }^{1}$ \\ Marcos Jesús Iglesias Martínez ${ }^{2}$ \\ INÉS LOZANO CABEZAS ${ }^{3}$
}

\begin{abstract}
RESUMEN: El trabajo colaborativo es un proceso con especial potencial innovador basado en las comunidades profesionales de aprendizaje, por lo tanto resulta importante conocer si las ideas del alumnado se corresponden con las características específicas de esta estrategia. En el presente artículo, se describió y analizó la valoración del trabajo colaborativo desarrollado por un grupo de maestros españoles. Los resultados muestran que los participantes tienen una concepción óptima del trabajo colaborativo, pero no se detecta que el alumnado lo perciba como una competencia profesional docente. Estas aportaciones tienen especial repercusión en el escenario internacional y más específicamente en la comunidad educativa latino americana, donde se exploran las oportunidades para la renovación de la práctica educativa en los niveles formativos iniciales.
\end{abstract}

Palabras clave: Educación superior. Formación inicial del profesorado. Colaboración. Competencias. Comunidades profesionales de aprendizaje.

\section{COLLABORATIVE WORK IN HIGHER EDUCATION: A PROFESSIONAL COMPETENCE FOR FUTURE PROFESSORS}

ABSTRACT: Collaborative work is a process with a special new potential based on professional learning communities, and thus it is important to understand if students' ideas correspond to the specific characteristics of this strategy. In this paper, we have described and analyzed the appraisal of collaborative work in a group of teachers from

\footnotetext{
*Parte de los datos de este trabajo pertenecen al proyecto de investigación "Cómo potenciar el desarrollo de la competencia una mirada profesional en la formación inicial”, financiado por la Conselleria d'Educatión, Investigación, Cultura i Esport de la Generalitat Valenciana (España). Referencia: GV/2014/075.

${ }^{1}$ Universidad de Oviedo, Facultad de Formación del Profesorado y Educación, Asturias, España.

${ }^{2}$ Universidad de Alicante, Facultad de Educación, Alicante, España. E-mail: marcos.iglesias@ua.es

${ }^{3}$ Universidad de Alicante, Facultad de Educación, Alicante, España.

DOI: 10.1590/ES0101-73302016147914
} 
Spanish universities. The results demonstrate that the participants have a very good understanding of this methodology, but it was not found that the students consider it as a professional teaching skill. These aspects have particular repercussions on the international teaching community, and more specifically on the Latin-American teaching community, where opportunities for the reform of teaching practices should be examined in the pedagogical practice in initial training levels.

Keywords: Higher education. Initial teacher education. Collaboration. Competences. Professional learning communities.

\section{LE TRAVAIL COLLABORATIVE DANS L'ÉNSEIGNEMENT SUPERIEUR: UNE COMPÉTENCE PROFESSIONNELLE POUR LES FUTURS ENSEIGNANTS}

$R E S U M E$ : Le travail collaboratif est un processus avec un fort potentiel dans le développement de communautés d'apprentissage professionneles, donc il est aussi important de comprendre les idées des élèves sur cette stratégie. Dans cet article nous décrivons et analysons l'évaluation de cette méthodologie par les professeurs d'université espagnole. Le résultat montre que les participants ont une bonne compréhension de cette méthodologie, bien que les étudiants ne considèrent pas cela comme une compétence professionnelle essentielle. Ces aspects ont un impact particulier sur, en particulier la communauté internationale de l'éducation en Amérique latine, où les possibilités de renouveler les pratiques d'enseignement sont spécialement mises en valeur.

Mots clés: Éducation Supérieure. Formation Initiale des Enseignants. Collaboration. Compétence. Communautés d'apprentissage Professionnelles.

\section{Introducción}

$\mathrm{L}$ a educación universitaria europea, más concreto la espańola, está sufriendo alteraciones sobre las concepciones del aprendizaje de los estudiantes universitarios y, especialmente, en el diseño de las estrategias metodológicas de los currículos de la educación superior, basadas fundamentalmente en la configuración de competencias profesionales y en las perspectivas socio-culturales como base para la formación de los estudiantes universitarios. Desde las perspectivas internacionales en el que lo delineamiento de lo que los profesores han de conocer y ser capaces de hacer (COCHRAN-SMITH et al., 2008; DARLING-HAMMOND, 2005; BRANSFORD, 2005; PERRENOUD, 2004), resaltase la necesidad de conocer el concepto de colaboración. 
En este sentido, Darling-Hammond (2008) argumenta la necesidad de que el profesorado conozca y comprenda los beneficios de la colaboración, porque ello supondrá conocer las interacciones entre sus estudiantes para potenciar el aprendizaje compartido entre ellos. Los docentes necesitan entender las prácticas, los diseños y los resultados de las estrategias colaborativas, dado que esto supone mejora del aprendizaje desde dentro y fuera de las escuelas, incluyendo a las familias como componentes fundamentales en la comunidad educativa, especialmente en los niveles formativos iniciales (IGLESIAS; LOZANO; MARTÍNEZ, 2013). Además, los maestros que trabajan en culturas colaborativas adquieren y desarrollan actitudes más positivas hacia la enseńanza (FLORES; DAY, 2006). Como afirman Hargreaves et al. (2001), es necesario formar docentes para generar una cultura profesional de relaciones constructivas y de este modo crear redes de aprendizaje fuertes e inteligentes para alcanzar propósitos en conjunto.

No sólo se ha de considerar el trabajo colaborativo, sino la importancia de la investigación colaborativa basada en la responsabilidad social en la acción educativa. Cochran-Smith y Lytle (2009) defienden este tipo de investigación entre los miembros de la comunidad educativa. El concepto de comunidad y colaboración abordado por las autoras es considerado una oportunidad de cambio para la comunidad educativa que trabaja e investiga colaborativamente (COCHRAN-SMITH; LYTLE, 2011). Los docentes son la conciencia educativa de la sociedad y, por lo tanto, han de ser expertos de colaboración mutua (MARINA, 2011), porque la educación es una responsabilidad colectiva que implica a toda la comunidad educativa (GOODLAD, 1984).

El aprendizaje del profesorado ha de consolidarse en base a sus propias experiencias formativas pedagógicas y didácticas, para luego construir sus propias teorías desde la práctica (KORTHAGEN, 2010). Para Kennedy (2011), el aprendizaje colaborativo es un aspecto positivo y eficaz para el desarrollo profesional docente. La importancia de "mirar profesionalmente" (MASON, 2002) es un elemento esencial en la formación de maestros, lo que sólo es posible a través de las experiencias prácticas de los futuros docentes por medio de una auténtica guía de observación personal. Grossman et al. (2009) establecen que las prácticas compartidas de los estudiantes mantienen la integridad y la complejidad en el aprendizaje de la enseñanza como un elemento nuclear en la formación docente. Asimismo, Nóvoa (2009) resalta la necesidad de profundizar en los equipos pedagógicos para consolidar la existencia de prácticas de colaboración, las cuales no se imponen por la administración, sino que se refuerzan en las comunidades de práctica y éstas han de ser desarrolladas en los programas de formación del profesorado y durante los primeros años de ejercicio de los mismos.

Actualmente, las teorías de enseñanza y aprendizaje de corte práctico, basadas en la participación del alumnado en la práctica, especialmente en un contexto social, están inundando los planes de estudio universitarios, principalmente 
en la necesidad de crear espacios compartidos y metodologías participativas que favorezcan situaciones de aprendizajes colaborativos (JOHNSON; JOHNSON, 2009). Los diseños se centran en que sean desarrolladas competencias basadas en las destrezas metodológicas que propicien un aprendizaje activo y responsable en el estudiante, en un entorno de trabajo colaborativo. Hay que tener en cuenta las potencialidades del trabajo cooperativo para la formación y orientación del profesorado (GOIKOETXEA; PASCUAL, 2002). De este modo, las comunidades de aprendizaje son fundamentos para plantear interrogantes entre los miembros, con la intención de desarrollar un diálogo reflexivo con los otros miembros del equipo sobre la práctica en la que actúan (McLAUGHLIN; TALBERT, 2006).

Montero (2011) plantea la necesidad de que la colaboración y la coordinación entre el profesorado sea una actitud y una capacidad que se adquiera por los futuros maestros, basada en la confianza, el apoyo mutuo, la auto-revisión y el aprendizaje compartido profesional, para pasar de una cultura balcanizada a una más colaborativa (HARGREAVES; FULLAN, 2012). Por ello queremos insistir en la importancia de diseñar y desarrollar metodologías más participativas, fundamentadas en la comunidad práctica donde el conocimiento es situado, colaborativo y reiterativo (HUBER, 2008), pues este tipo de metodología favorece la participación y la intercomunicación entre el alumnado, y aumenta su responsabilidad compartida.

Tras la exhaustiva revisión de la literatura científica, se destacan la importancia y la necesidad de indagar en este tipo de investigaciones la formación de los docentes que trabajan en grupos de colaboración para la mejora de la enseñanza y del aprendizaje en las escuelas (WONG et al., 2014). Por lo tanto, el reto que se planteaba y dio la génesis de esta investigación se centraba en conocer y analizar las concepciones de los estudiantes de magisterio sobre el trabajo colaborativo y en investigar sus experiencias prácticas acerca de esta competencia profesional que ha de adquirir un futuro docente para la mejora de la calidad de la educación a través de la creación de comunidades profesionales de aprendizaje.

\section{El estudio}

\section{Contexto y desarrollo del proceso}

Esta experiencia se llevó a cabo durante el curso 2012/2013 en la asignatura de "Diseño de los Procesos Educativos", perteneciente al primer curso del Grado de Maestro de Educación Primaria de la Universidad de Alicante, con una carga de seis créditos en el Sistema Europeo de Transferencia y Acumulación de Créditos (ECTS), siendo 1.5 de formación teórica y 4.5 de actividades prácticas. Los suyos contenidos suponen adquirir estrategias y competencias profesionales docentes en el diseño de los procesos de enseñanza-aprendizaje. 
El carácter práctico de esta asignatura así como lo contenidos trabajados hacen posible desarrollar distintas metodologías en el aula, entre ellas el trabajo colaborativo. Es importante destacar que el alumnado de primer curso nos permite descubrir tempranamente las necesidades formativas en cuanto a metodología se refiere, para de este modo diseñar aspectos formativos que puedan obtener en cursos posteriores durante el Grado que cursan, y aplicarlos en las prácticas docentes de las escuelas de primaria.

Para lograr los objetivos propuestos en el programa de la asignatura, se les planteó a los estudiantes elaborar las prácticas académicas mediante grupos heterogéneos de trabajo colaborativo, los cuales eran formados por cuatro o cinco personas, con variedad de edad, género y origen en sus estudios previos a la universidad. Esto posibilitó que en tales agrupaciones mantuviesen una variedad rica en aportaciones con puntos de vista diversos y perspectivas en ocasiones encontradas. El grupo multidisciplinar diseñó cinco fases de información y concienciación sobre cada una de las partes de la investigación.

En primer lugar, y tomando como base la noción de comunidad del aprendizaje, se les explicó el concepto del trabajo colaborativo o cooperativo como un conjunto de personas autónomas y reflexivas que trabajan en armonía y en apoyo mutuo y buscan el desarrollo de profesionales de la educación comunicándose entre ellos. En segundo lugar, se les aconsejó la utilización de diferentes recursos para que el desarrollo de esta metodología fuera más eficaz. En tercer lugar, se les aconsejó que la cumplimentación de un acta de trabajo como recurso útil para ayudar a los maestros a reflexionar y a mejorar el trabajo realizado. En cuarto lugar, se les recomendó el empleo de las herramientas digitales, especialmente de las redes sociales y plataformas digitales al desarrollo del trabajo y la puesta en común de los objetivos alcanzados. Finalmente, en quinto lugar, se les orientó para reflexionar sobre su desarrollo en el trabajo colaborativo que tuviesen como base la conversación evaluativa, con el objetivo de plantear ideas y explicaciones, fomentar el diálogo reflexivo, permitir la evaluación de las mismas ideas y sintetizar como grupo las decisiones consensuadas.

\section{Objetivos de la investigación}

Para el análisis de esta investigación se plantean los siguientes objetivos:

- Conocer las concepciones que tienen los estudiantes del Grado de Maestro de Educación Primaria sobre el trabajo colaborativo, así como las herramientas y los recursos que utilizan en el desarrollo del mismo.

- Conocer y analizar los beneficios y las dificultades del trabajo colaborativo como metodología de aprendizaje para la adquisición de competencias profesionales. 
- Identificar las posibles necesidades formativas en relación al trabajo colaborativo como competencia profesional.

\section{Participantes}

Se ha seleccionado una muestra para esta investigación de 83 alumnos, futuros docentes matriculados en el Grado de Maestro de Educación Primaria, con edades comprendidas entre 18 a 35. Se han excluido, por diversos motivos de fiabilidad, 25 sujetos, quedando un total de 58 ( $73 \%$ mujeres y $27 \%$ hombres). La mayoría de los participantes proviene de los estudios de bachillerato (61.31\%), el resto de la formación profesional $(34,29 \%)$ u otras vías de acceso como, por ejemplo, mayores de 25 años $(4,40 \%)$.

\section{Recogida y análisis de datos}

Se diseñó un cuestionario estructurado en dos partes: la primera plantea seis cuestiones abiertas sobre el concepto, la valoración y la utilización de distintos recursos o herramientas para llevar a cabo el trabajo colaborativo; mientras en la segunda, se diseña para responder a 11 preguntas cerradas tipo escala Likert, a las que se les da un valor numérico del uno al quinto asignándole un valor descriptivo con la siguiente correspondencia: $1=$ totalmente en desacuerdo, 2 = desacuerdo, $3=$ ni de acuerdo, ni en desacuerdo, $4=\mathrm{de}$ acuerdo y $5=$ totalmente de acuerdo. El cuestionario redactado inicialmente es validado por tres expertos del área de Didáctica y Organización Escolar de dos universidades (Universidad de Alicante y Universidad de Oviedo), a partir de cuyas orientaciones se confecciona el cuestionario definitivo. Aquello se cumplimenta al finalizar la asignatura de manera anónima y voluntaria. Se procede a una breve presentación oral de la propuesta y del objetivo de la investigación y se puntualiza las cuestiones o dudas al respecto.

El tratamiento de los datos se realiza con el apoyo de dos soportes informáticos: AQUAD 6 (HUBER; GÜRTLER, 2012), por lo cual se analizan las narrativas de los estudiantes relativas a las seis cuestiones abiertas del cuestionario; y a través del programa SPSS versión 19, es hecho un análisis de la parte cerrada para obtener finalmente una valoración global del trabajo colaborativo.

A partir de la muestra detallada se opta por una metodología mixta, pero con alto contenido cualitativo, de ahí que en el proceso de codificación se produzca una saturación de los códigos emergentes, por lo que no ha sido necesario ampliar la muestra. En este sentido, el proceso del análisis de datos cualitativos transcurre mediante el enfoque mixto (deductivo-inductivo), lo que permite configurar el mapa de categorías, útil para el proceso de codificación. Estos mapas se 
analizan y validan desde una perspectiva pluri-institucional (entre la Universidad de Alicante y la Universidad de Oviedo) por tres equipos de expertos del área de Didáctica y Organización Escolar. Se consolida, posteriormente, con alguna modificación menor como consecuencia de los ajustes derivados de la intensidad de la codificación y de las posibles variantes o matizaciones emergentes y en relación al marco conceptual establecido (GLASER; STRAUSS, 1967), para lograr una compresión más profunda del fenómeno de estudio (MILES; HUBERMAN, 1994), respetando en todo momento las prescripciones propias de la metodología cualitativa (CORBIN; STRAUSS, 2008). Las unidades de codificación se identifican por un sistema alfanumérico para garantizar el anonimato de los participantes, siendo representado por Alu000.

\section{Presentación y análisis de resultados}

Siguiendo la estructura del estudio, se presenta un análisis de los resultados en dos partes. En primer lugar, exponemos y analizamos los datos obtenidos de las respuestas abiertas (parte cualitativa de la investigación); donde mediante las Tablas 1 hasta 5 son mostradas las categorías y los códigos resultantes, conociendo de este modo el concepto que tienen los participantes sobre el trabajo colaborativo, así como los beneficios, las dificultades, los roles establecidos y los recursos utilizados para el desarrollo de esta metodología como competencia profesional docente. Para la exposición de los resultados, se utilizan tablas que agrupan los códigos en cada una de las categorías con sus diferentes frecuencias de aparición $(\mathrm{FA} \%)$ y la frecuencia relativa (FR) sobre el total de participantes (cuyo cálculo es resultado de la formula FA/n, siendo $\mathrm{n}=58$ ). En segundo lugar, en la Tabla 6, se exponen los resultados referidos a la parte cuantitativa de la investigación, analizando las respuestas cerradas para la valoración del trabajo colaborativo a través de la media, la desviación típica (DT) y el porcentaje de los participantes que han otorgado los valores más altos.

Tabla 1

Análisis del concepto de trabajo colaborativo.

\begin{tabular}{l|c|c}
\hline Códigos & FA (\%) & FR \\
\hline 1.1 Cooperación-participación activa & 46,02 & 1,40 \\
\hline 1.2 Interacción social & 34,09 & 1,03 \\
\hline 1.3 Objetivos comunes & 17,05 & 0,52 \\
\hline 1.4 Producción eficaz y calidad & 2,27 & 0,07 \\
\hline 1.5 Competencia profesional & 0,57 & 0,02 \\
\hline
\end{tabular}

FA: frecuencia de aparición; FR: frecuencia relativa. 
Tabla 2

Análisis de los beneficios del trabajo colaborativo.

\begin{tabular}{l|c|c}
\hline Códigos & FA $\mathbf{( \% )}$ & FR \\
\hline 2.1 Interacción social-conocimiento & 51,92 & 1,40 \\
\hline 2.2 Reparto de tareas equitativo & 18,59 & 0,50 \\
\hline 2.3 Coadyuvar y aprender & 16,67 & 0,45 \\
\hline 2.4 Producción eficaz en trabajo & 12,18 & 0,33 \\
\hline 2.5 Competencia profesional & 0,64 & 0,02 \\
\hline
\end{tabular}

$F A$ : frecuencia de aparición; $F R$ : frecuencia relativa.

Tabla 3

Análisis de las dificultades del trabajo colaborativo.

\begin{tabular}{l|c|c}
\hline Códigos & FA (\%) & FR \\
\hline 3.1 Falta de consenso & 40,54 & 0,78 \\
\hline 3.2 Falta de compromiso & 25,23 & 0,48 \\
\hline 3.3 Falta de tiempo & 21,62 & 0,41 \\
\hline 3.4 Otros problemas & 9,91 & 0,19 \\
\hline 3.5 No hay dificultades & 2,70 & 0,05 \\
\hline
\end{tabular}

FA: frecuencia de aparición; FR: frecuencia relativa.

Tabla 4

Roles adoptados en el trabajo colaborativo.

\begin{tabular}{l|c|c}
\hline Códigos & FA (\%) & FR \\
\hline 4.1 Ausencia de roles & 78,26 & 0,93 \\
\hline 4.2 Coordinador/a & 4,35 & 0,05 \\
\hline 4.3 Secretario/a o redactor/a & 7,25 & 0,09 \\
\hline 4.4 Responsable de búsqueda de la información & 10,14 & 0,12 \\
\hline
\end{tabular}

FA: frecuencia de aparición; FR: frecuencia relativa.

Tabla 5

Análisis de los recursos utilizados en el trabajo colaborativo.

\begin{tabular}{l|c|c}
\hline Códigos & FA (\%) & FR \\
\hline 5.1 Herramientas de la telecomunicación digital & 52,71 & 1,34 \\
\hline 5.2 Actas de trabajo para la reflexión & 20,27 & 0,52 \\
\hline 5.3 Actas de trabajo al control del profesorado & 15,54 & 0,40 \\
\hline 5.4 Actas de trabajo para la planificación & 11,49 & 0,29 \\
\hline
\end{tabular}

FA: frecuencia de aparición; FR: frecuencia relativa. 


\section{Tabla 6}

Valoración global del trabajo colaborativo.

\begin{tabular}{l|c|c|c}
\hline Ítems & Media & DT & DA-TA (\%) \\
\hline 1. Ha mejorado la interacción entre compańeros. & 4,14 & 0,88 & 77,59 \\
\hline 2. He aprendido a respetar las opiniones de mis compañeros. & 4,26 & 0,71 & 84,48 \\
\hline 3. He aprendido a defender y argumentar mis opiniones. & 4,12 & 0,82 & 72,41 \\
\hline $\begin{array}{l}\text { 4. Nos ha ayudado a planificarnos con tiempo para realizar } \\
\text { los trabajos. }\end{array}$ & 3,38 & 0,83 & 41,38 \\
\hline 5. La eficacia del trabajo en grupo ha sido satisfactoria. & 3,81 & 1,02 & 67,24 \\
\hline $\begin{array}{l}\text { 6. Todos los miembros del grupo han participado } \\
\text { activamente casi todo el tiempo. }\end{array}$ & 3,52 & 1,03 & 50,00 \\
\hline $\begin{array}{l}\text { 7. Me ha ayudado asimilar mejor los contenidos de la } \\
\text { asignatura. }\end{array}$ & 3,93 & 0,81 & 70,69 \\
\hline $\begin{array}{l}\text { 8. Estoy satisfecho con los resultados obtenidos al trabajar } \\
\text { colaborativamente. }\end{array}$ & 4,10 & 0,85 & 81,03 \\
\hline $\begin{array}{l}\text { 9. Ha faltado coordinación, tener los roles claros dentro del } \\
\text { grupo de trabajo. }\end{array}$ & 2,41 & 1,14 & 17,24 \\
\hline $\begin{array}{l}\text { 10. Me considero responsable de los resultados de la práctica } \\
\text { realizada colaborativamente. }\end{array}$ & 3,79 & 0,95 & 58,62 \\
\hline $\begin{array}{l}\text { 11. Considero que trabajar colaborativamente es esencial en } \\
\text { la formación de los/as maestros/as. }\end{array}$ & 4,66 & 0,58 & 94,83 \\
\hline
\end{tabular}

DA: de acuerdo; TA: totalmente de acuerdo; DT: desviación típica.

\section{Parte cualitativa}

En la Tabla 1 se recogen los resultados referidos a las concepciones de los estudiantes sobre el trabajo colaborativo.

Es necesario conocer la percepción que tienen los estudiantes del concepto, de los beneficios y de las dificultades del trabajo colaborativo, así como la manera de llevar a cabo el proyecto con los recursos aportados, aplicando esta metodología. De este modo, comprobaremos si es necesario trabajar algún aspecto en concreto y de qué manera para conseguir que no solo se trate de una metodología de aula, sino de una competencia profesional para nuestros futuros docentes.

Por ello, lo primero que se les plantea a los participantes es que piensen y definan el concepto de trabajo colaborativo, de esta reflexión surge que la colaboración y la participación activa de todos los miembros del grupo (código 1.1) son fundamental para la mayoría de los participantes. En este sentido, son muy numerosas las narrativas que hacen referencia a esta característica fundamental del trabajo colaborativo como metodología de aprendizaje: 
Aquel en el que todos los integrantes participan de forma colaborativa, den sus opiniones y respeten las de los demás (Alu038).

Un trabajo en que todas las componentes del grupo participan activamente y conjuntamente (Alu044).

Por otra parte, los relatos de los estudiantes hacen énfasis en la importancia de la interacción social, fundamentada en el respeto y en la escucha activa, y basada principalmente en poner en común ideas desde el dialogo para lograr una mejor comunicación y un entendimiento en el desarrollo de las acciones (código 1.2):

Consiste en trabajar con otras personas, respetando y que respeten mis opiniones para llegar a un acuerdo (Alu029).

Ayudándonos unos a otros para realizar un trabajo, mejorar las interacciones entre unos y otros (Alu047).

Los aspectos que hemos mencionado son atributos específicos del trabajo colaborativo. Sin embargo, los estudiantes también coinciden que, para el desarrollo de una tarea cooperativa, es necesario clarificar un objetivo común, saber hacia dónde dirigir el proyecto, y esto se consigue después de una buena coordinación. El alumnado tiene claro que cuando ambos factores fluyen sin complicaciones se consigue llegar a un objetivo común (código 1.3):

El trabajo colaborativo es aquel que se realiza colectivamente y está orientado hacia unos objetivos comunes (Alu051).

Es la cooperación de un grupo de personas para lograr unos fines comunes, como un proyecto teórico o práctico (Alu052).

Aunque son escasas las narrativas que consideran que el trabajar colaborativamente hace que las producciones tengan más calidad (código 1.4), algunos participantes han hecho alusión a ello:

Es aquel que se realiza conjuntamente con compañeros, cosa que ayuda a enriquecer el trabajo y, desde luego, a mejorar sus resultados con la ayuda de todos (Alu031).

Solo se ha localizado un relato en el que se describe el concepto de trabajo colaborativo como una competencia profesional que ha de adquirir el futuro maestro (código 1.5 - Competencia profesional):

El trabajo colaborativo es una metodología que han de conocer todos los maestros para trabajar en la escuela (Alu029). 
En suma, se puede afirmar que el alumnado considera que el trabajo colaborativo se basa en llevar a cabo un proyecto común, en el que todos los participantes colaboren de manera activa aportando ideas mediante una buena interacción social.

En relación a los beneficios que reportan las acciones cooperativas, los resultados están aglutinados en la Tabla 2.

Como hemos hecho referencia con anterioridad, los participantes consideran que el trabajo colaborativo se basa en una óptima interacción social (código 2.1). Numerosas narrativas hacen referencia a ello cuando se les ha preguntado por los beneficios de esta metodología. Además de destacar la importancia de las relaciones con los demás participantes, el alumnado considera que esta práctica cooperativa permite crear un conocimiento compartido entre los miembros y enriquecerse gracias a las opiniones de los otros:

Que aprendemos diferentes técnicas grupales y podemos ver las cosas desde diferentes puntos de vista (Alu054).

Pues las ventajas serían la interacción entre los compañeros, conocerlos, compartir opiniones, pasar más tiempo juntos/as etc (Alu039).

Por otra parte, los estudiantes manifiestan que el trabajo colaborativo permite un reparto más equitativo de las tareas y genera la posibilidad de trabajar según las habilidades o destrezas de cada miembro del grupo (código 2.2):

Para mí, lo más positivo es el hecho de que, si el trabajo es denso, cada miembro del grupo puede encargarse de algo, repartiendo así el esfuerzo (Alu032).

Se adapta cada una de ellas a las características de cada sujeto (Alu002).

La reflexión acerca de los beneficios del trabajo colaborativo y la puesta en marcha de la metodología han hecho que los estudiantes consideren que un aprendizaje en cooperación, mediante la interacción con los compañeros, ayuda a comprender mejor los problemas y los contenidos a trabajar (código 2.3):

Te puedes ayudar a resolver dudas o cosas que no tengas claras con la ayuda de los compañeros (Alu021).

Bajo mi punto de vista, [el trabajo colaborativo] tiene una ventaja esencial: completar las carencias de información. Es decir, ayuda a aprender de los demás (Alu031).

Con el objetivo de conocer las posibles dificultades u obstáculos en el desarrollo del trabajo colaborativo, se le solicitó al alumnado que reflexionara sobre las posibles problemáticas detectadas al desarrollar esta metodología de aprendizaje. En la Tabla 3 se muestran los resultados de las codificaciones. 
Una de las claves del trabajo colaborativo, como mencionado con anterioridad, son la coordinación y la organización para conducir un proyecto común con éxito, pero a su vez es el elemento de esta metodología que entrańa mayor dificultad para el alumnado. El momento de diseńar las acciones para el desarrollo de una tarea conlleva ausencia de consenso para la toma de decisiones, desacuerdos en la organización y planificación o, simplemente, descoordinación en el transcurso del trabajo (código 3.1):

$\mathrm{El}$ inconveniente que tiene es que en ocasiones surgen disputas entre los compańeros, por no estar en acuerdo en diversas opiniones, o por no compartir el comportamiento del otro (Alu006).

A veces los puntos de vista son demasiados opuestos y no se llega fácilmente a un consenso (Alu034).

A la falta de coordinación y organización como dificultades descritas por el alumnado, se suma la falta de responsabilidad y compromiso en la elaboración de las tareas (código 3.2):

Dificultades: el trabajo colaborativo debe adaptarse al grupo, no al individuo (Alu016).

La falta de colaboración en algunas cosas (Alu033).

La filosofía del plan Bolonia ha provocado en el alumnado universitario la responsabilización por una importante carga de trabajo práctico, lo que conlleva dificultades para la planificación en sus espacios y horarios en el desarrollo de los proyectos de las asignaturas cursadas (código 3.3):

Dependes de la disponibilidad de los miembros y se hace poco difícil el poder quedar a determinadas horas (Alu010).

El único inconveniente es que en ocasiones resulta difícil quedar por los diferentes horarios o zonas de residencia (Alu035).

Con menor frecuencia, se han detectado otros problemas que surgen en la dinámica del trabajo (código 3.4):

Tienes que amoldar a todos los compañeros y su modo de trabajo (Alu043).

También se han hallado algunos relatos en los que se afirman no existir dificultades:

Ninguno. Lo importante es que se trabaje en grupo, dialogando y colaborando todos por igual, para que el trabajo en grupo funcione bien (Alu054). 
En las entrevistas el alumnado reflexionó sobre los roles asumidos durante el desarrollo de su trabajo colaborativo. Como resultado del mismo, en la Tabla 4 se agrupan el rol adoptado más las descripciones de las tareas desarrolladas en el mismo.

Los estudiantes, en su mayoría, consideran que no han asumido un rol específico, sino, más bien, que el desarrollo de las funciones ha sido mediante un consenso y reparto equitativo del trabajo:

Todos en el grupo hemos hecho de todo por consenso, por igual nos repartíamos las funciones, pero todos hacíamos de coordinadores, búsqueda de información, todos participábamos en pasarlo al ordenador y dar formato, todos implicados para hacer un buen trabajo (Alu010).

Se observó, por otra parte, la existencia de narrativas identificando que algunos compañeros han desarrollado funciones como coordinador/a del grupo "sí, normalmente tomo el mando en el grupo, coordinando a los demás" (Alu049); secretario/a del grupo de trabajo - "el mío, en concreto, ha sido el de redactora o secretaria del grupo" (Alu032); o responsable de búsqueda y localización de la información - "he sido la encargada de búsqueda de información, pero nunca de coordinadora, porque no va con mi forma de ser y no sé me da bien" (Alu048).

Las telecomunicaciones digitales son una práctica habitual entre el alumnado universitario, ya que en el diseño y la elaboración de cualquier práctica académica viene implícita el hecho de la utilización de las mismas, sea para la comunicación entre los miembros del grupo (redes sociales, chat, foros de discusión etc.), el desarrollo de las tareas en red (Google Drive) o la búsqueda y localización de la información (código 5.1):

Además de otras redes sociales, principalmente el email (Alu042).

Hemos utilizado recursos extraídos del Google como el Google Drive para trabajar en red (Alu052).

Como material complementario se les sugirió que realizaran un acta de trabajo para recoger el desarrollo de la práctica, con el propósito de fomentar un diálogo reflexivo entre los miembros del grupo; para posteriormente preguntarles por las utilidades de la misma. De los relatos analizados, la mayoría del alumnado ha considerado la utilización del acta de trabajo un instrumento para valorar y plantear propuestas de mejora en la elaboración del trabajo (código 5.2):

Es una buena manera de plantear los problemas que han ocurrido durante el tiempo de trabajo común (Au014).

Sirve para conocer cómo funciona el grupo y poder ir haciendo un seguimiento de su evolución (Alu031). 
Dado que la propuesta de la cumplimentación del acta de trabajo surge por parte del profesorado, algunos estudiantes consideran que este recurso se propone con la finalidad de controlar el trabajo realizado por los miembros del grupo. De este modo, la autoevaluación pretendida se entiende como una evaluación por parte del profesorado de la asignatura hacia el alumnado. Así se recoge en el código 5.3:

La utilidad del acta de trabajo sirve más para el profesor, para controlar lo que hacemos cada uno. Para nosotros no nos sirve mucho (Alu026).

Una forma de controlar la práctica, de reflejar los datos del grupo (Alu012).

No obstante, y en contraposición a lo anterior, algunas narrativas consideran el acta de trabajo como una herramienta útil para la planificación de las tareas, como queda expresado en el siguiente fragmento (código 5.4):

Yo creo que las actas nos ayudan a organizarnos (Alu021).

\section{Parte cuantitativa}

Una vez cumplimentada la entrevista abierta, los estudiantes respondieron a un cuestionario cerrado para contrastar los datos en la investigación y complementar los resultados obtenidos. La Tabla 6 muestra los resultados hallados en torno a 11 ítems diferenciados, recogiendo las valoraciones del alumnado sobre el trabajo colaborativo. Para este análisis, se han tomado como prioridad los datos estadísticos que describen la muestra, centrándonos en la media resultante de los valores obtenidos entre los participantes, la DT de los valores para obtener una perspectiva grupal y el porcentaje de los participantes que han otorgado los valores cinco y cuatro ( $\mathrm{TA}=$ totalmente de acuerdo y DA = de acuerdo) a cada uno de los ítems.

Observamos que la mayoría de las medias obtenidas de los ítems propuestos se encuentran por encima del valor tres y acercándose al cuatro (DA). La DT alcanza un valor bajo lo que significa que los datos obtenidos son muy homogéneos, esto lo podemos comprobar a su vez mediante los porcentajes hallados en la tercera columna que hacen referencia al alumnado que considera estar DA o TA con los ítems planteados en el cuestionario.

Los datos muestran que la interacción social de manera óptima, es decir, respetando las opiniones, escuchando activamente, defendiendo y argumentando ideas, propuestas, soluciones etc., (ítems 1,2 y 3 ) es valorada muy positivamente por el alumnado al desarrollar este tipo de metodologías en el aula. Es importante destacar que los participantes consideran que todos los miembros del grupo han 
participado activamente en el trabajo (ítem 6) y que la colaboración entre todos les ha ayudado a asimilar mejor los contenidos de la asignatura (ítem 7). Todo ello ha llevado a que el alumnado esté satisfecho en su mayoría con el resultado final de su proyecto (ítem 8) y con la eficacia de la metodología empleada (ítem 5).

Al contrario de los resultados cualitativos (Tabla 1), en el que los participantes no hacían alusión a la competencia profesional que les podía aportar al trabajar este tipo de metodología de aprendizaje. En esta segunda fase de la investigación, se les pregunta explícitamente sobre si el trabajo colaborativo es esencial para la formación docente (ítem 11), y un alto porcentaje de los estudiantes lo consideran como un aspecto fundamental.

Por otra parte, en el ítem 9 se observa que el porcentaje de participantes que están de acuerdo con esta afirmación es muy bajo, esto se contradice con los resultados obtenidos con la pregunta abierta (Tabla 4) en la que afirmaban trabajar sin adoptar roles. Ellos tienden a repartir las tareas de manera equitativa y ello les hace sentirse en todos momentos responsables del resultado final (ítem 10).

\section{Discusión y conclusiones}

Hemos podido detectar que para la mayoría del alumnado trabajar colaborativamente conlleva la coordinación y la cooperación entre los miembros de un equipo, potenciando la interacción social basada en el diálogo o la escucha de las opiniones de los demás, teniendo como finalidad la consecución de unos objetivos comunes. Coincidimos con Cochran-Smith y Lytle (2011) que un elemento clave del trabajo colaborativo es la reflexión de todos los miembros que trabajan en colaboración dentro de una comunidad profesional de aprendizaje. Sin embargo, hemos de resaltar que esta no se ha tenido en cuenta en ninguna de las descripciones de los participantes. Por ello, consideramos importante tratar de que el alumnado potencie y desarrolle la capacidad de diálogo reflexivo como característica relevante en este tipo de metodología dentro de las clases universitarias, especialmente en la formación de los maestros.

Otro de los hallazgos importantes en esta investigación es la concepción que tienen los participantes sobre el trabajo colaborativo, la cual se queda desvinculada de asumir esta metodología como competencia profesional docente. Así, en el análisis de los beneficios, tan solo nos encontramos con una narrativa que considera favorable esta adquisición para su profesión, aspecto que coincide con la investigación realizada por Dobber et al. (2013). Sin embargo, en el análisis de los datos cuantitativos, hemos detectado que la mayoría de los participantes afirman estar de acuerdo con la adquisición de esta competencia. Esta contradicción entendemos que se puede deber a que sus pensamientos iniciales están orientados a asumir esta metodología como una herramienta que les facilita el aprendizaje de 
los contenidos de la asignatura, pero no son conscientes de los objetivos de esta dinámica de trabajo como competencia profesional, hasta que no se les plantea en el cuestionario cerrado que se les proporciona en una segunda fase.

Las dificultades detectadas por los participantes se identifican, principalmente, con las actitudes y conductas de cada miembro del grupo. Se destaca, entre otras, la falta de responsabilidad y compromiso individual para que el trabajo colaborativo sea efectivo. Asimismo, es necesaria la plena coordinación y la planificación de las tareas, que de manera conjunta y junto con los esfuerzos individuales y compartidos, pueda contribuir a la consecución de los objetivos del equipo. Por lo tanto, las tareas y las actividades han de estar inundadas por una interacción comunicativa continua, con el propósito de que el trabajo sea eficaz, conclusiones que coinciden con el estudio de Crichton y Templeton (2013).

En el análisis de los recursos utilizados, consideramos que no ha existido una utilización óptima de la diversidad de herramientas que pueden aportar las telecomunicaciones digitales, y como han evidenciado otras investigaciones en la Educación Superior y, en especial en la formación del profesorado (IGLESIAS; LOZANO; MARTÍNEZ, 2013) y que hubiera evitado en parte, otra de las dificultades a la que los participantes han hecho alusión, como la falta de tiempo y de espacios. Tan solo en algunas ocasiones han utilizado los recursos digitales para la comunicación y búsqueda de información y para la elaboración del proyecto a través de la red, como nos permiten numerosas plataformas virtuales (Google Drive). La investigación llevada a cabo por Batarelo, Nevinand y Malian (2013) considera que los avances en la tecnología de información y comunicación son doblemente importantes, ya que están relacionados con las características que están disponibles en los entornos en línea; y también con los cambios en el conocimiento previo y la experiencia de la tecnología que los estudiantes aplican para el desarrollo del aprendizaje colaborativo.

Entre los recursos planteados a los participantes, se les sugirió la utilización de la elaboración del acta de trabajo como un instrumento que contribuye a la reflexión del trabajo colaborativo, como han evidenciado otras investigaciones (PALOMARES, 2011). Aunque los participantes de nuestro estudio no han utilizado el concepto de la reflexión, sí que han orientado este recurso hacia la autoevaluación para la mejora de su trabajo. No obstante, es significativo destacar que muchos de los participantes consideran todas las herramientas de trabajo como elementos evaluativos por parte del profesorado, transformando de este modo un instrumento de reflexión en una herramienta de control para ser ellos evaluados.

En relación al reparto de roles, la mayoría de los participantes afirma no haber adoptado ningún tipo específico, si no haber repartido el trabajo equitativamente. Esto puede ser debido a que el alumnado no ha sido formado con anterioridad en el sistema de reparto de roles (FORTE; FLORES, 2014), y para 
ellos la coordinación en grupo es repartir el trabajo ecuánimemente. En muchas ocasiones, hemos observado que el reparto de roles les ha supuesto más confusión y ha sido motivo de más discrepancias en el grupo que beneficios les haya podido aportar a la hora de trabajar colaborativamente.

Las conclusiones finales de este estudio nos permiten afirmar que el trabajo cooperativo entre los docentes puede contribuir a la mejora de la calidad de la docencia (DARLING-HAMMOND, 2008), tanto en los aspectos presenciales como en otros más virtuales (VÁZQUEZ; FOMBONA; FERNÁNDEZ, 2013). Además, consideramos que estas características se relacionan con las comunidades de práctica definidas por Wenger (1998), en el que se exigen relaciones simétricas y recíprocas a través de una negociación de nuevos significados y de aprendizajes entre los miembros de la comunidad. Por otra parte, estamos de acuerdo con Montero (2011) que esta metodología supone una oportunidad formativa excelente, basada en la responsabilidad colectiva, además de colaboración y participación espontánea y voluntaria para que se configuren las condiciones organizativas del trabajo colaborativo. De esta manera supondrá generar una cultura profesional docente de cooperación e interdependencia para la mejora de la práctica de la enseñanza y el aprendizaje en las escuelas, y en concreto a que los noveles puedan analizar y evaluar sus prácticas (KENNEDY, 2016).

Consideramos entonces la necesidad de potenciar culturas docentes colaborativas desde los programas de formación del profesorado, con la existencia de relaciones entre las escuelas y la universidad y, por lo tanto, la necesidad de crear y potenciar espacios híbridos, como propone Zeichner (2010), para que la calidad de programas de formación del profesorado sea mejor.

Se entiende el trabajo colaborativo como una competencia profesional que ha de adquirir el profesorado para potenciar la colaboración global e intercultural entre los miembros de un equipo docente que pretende alcanzar unos objetivos y compromisos compartidos, como auténticas comunidades profesionales de aprendizaje (OPFER; PEDDER, 2011; STOLL et al., 2006; VESCIO; ROSS; ADAMS, 2008). Se destaca el papel de cada individuo dentro de una comunidad de diálogo y reflexión, en el que todos los participantes contribuyen con su interacción a la resolución de algún problema o la consecución de los objetivos compartidos. Por lo tanto, existe la necesidad de diseñar programas de formación del profesorado basados en una cultura colaborativa, que integren: la interrelación social, el diálogo y la reflexión entre los docentes, la interacción entre teoría-práctica estudiada, las metodologías participativas basadas en trabajos por proyectos y la utilización de redes sociales para los profesionales de la enseñanza, incluyendo los recursos y medios que ofrecen las telecomunicaciones digitales. En suma, un programa de formación del profesorado en el que se integren la cognición individual y social, la comunidad de aprendizaje y el discurso reflexivo y compartido, que ofrezcan más garantías en la formación inicial docente. 


\section{Referencias}

BATARELO, I.; NEVIN, A.; MALIAN, I. Collaborative online course development: facilitation of multi-dimensional teaching and learning. Croatian Journal of Education, Croatian, v. 15, n. 2, p. 491-519, 2013.

COCHRAN-SMITH, M.; FEIMAN-NEMSER, S.; McINTYRE, D. J.; DEMERS, K. E. Handbook of Research on Teacher Education. Enduring Questions in Changing Contexts. New York: Routledge, 2008.

COCHRAN-SMITH, M.; LYTLE, S. Inquiry as stance: practitioner research for the next generation. New York: Teachers College Press, 2009.

. Changing Perspectives on Practitioner Research. Learning Landscapes, Canada, v. 4, n. 2, p. 17-23, 2011.

CORBIN, J.; STRAUSS, A. Basics of qualitative research. 3rd ed. Thousand Oaks, CA: SAGE, 2008.

CRICHTON, H.; TEMPLETON, B. Collaboration or confrontation? An investigation into the role of prior experiences in the completion of collaborative group tasks by student teachers. European Journal of Teacher Education, London, v. 36, n. 1, p. 84-96, 2013.

DARLING-HAMMOND, L.; BRANSFORD, J. (Eds.). Preparing teachers for a changing world: What teachers should learn and be able to do. San Francisco, CA: Jossey-Bass, 2005.

DARLING-HAMMOND, L. The case for university-based teacher education. In: COCHRAN, M. S.; FEIMAN-NEMSER, S.; McINTYRE, D. J.; DEMERS, K. E. (Eds.). Handbook of Research on Teacher Education. Enduring Questions in Changing Contexts. New York: Routledge, 2008. p. 333-346.

DOBBER, M.; VANDYCK, I.; AKKERMAN, S.; GRAAFF, R.; BEISHUIZEN, J.; PILOT, A. et al. The development of community competence in the teacher education curriculum. European Journal of Teacher Education, London, v. 36, n. 3, p. 346-363, 2013.

FLORES, M.; DAY, C. Contexts which shape and reshape new teachers' identities: A multi-perspective study. Teaching and Teacher Education, Amsterdam, v. 22, n. 2, p. 219232, 2006.

FORTE, A.; FLORES, M. Teacher collaboration and professional development in the workplace: a study of Portuguese teachers. European Journal of Teacher Education, London, v. 37, n. 1, p. 91-105, 2014.

GLASER, B. G.; STRAUSS, A. L. The discovery of grounded theory: strategies for qualitative research. New York: Aldine, 1967.

GOODLAD, P. A placed called school: prospects for the future. New York: McGraw-Hill, 1984.

GOIKOETXEA, E.; PASCUAL, G. Aprendizaje cooperativo: bases teóricas y hallazgos empíricos que explican su eficacia. Revista de Educación XX1, Madrid, v. 5, p. 227-247, 2002. 
GROSSMAN, P. Teaching practice: a cross-professional perspective. Teachers College Record, Columbia, v. 111, n. 9, p. 2065-2100, 2009.

KENNEDY, A. Collaborative continuing professional development (CPD) for teachers in Scotland: aspirations, opportunities and barriers. European Journal of Teacher Education, London, v. 34, n. 1, p. 25-41, 2011.

KENNEDY, M. Parsing the Practice of Teaching. Journal of Teacher Education, Michigan, v. 62 , n. 1, p. 6-17, 2016.

KORTHAGEN, F. La práctica, la teoría y la persona en la formación del profesorado. Revista Interuniversitaria Formación del Profesorado, Zaragoza, v. 68, p. 83-102, 2010.

HARGREAVES, A.; EARL, L.; MOORE, S.; MANNING, S. Learning to change. Teaching Beyond Subjects and Standards. San Francisco: Jossey Bass, 2001.

HARGREAVES, A.; FULLAN, M. Capital professional. Transforming Teaching in Every School. Toronto: Teachers College Press, 2012.

HUBER, G. Aprendizajes activos y metodologías educativas. Revista de Educación, Madrid, v. Extraordinario, p. 59-81, 2008.

HUBER, G.; GÜRTLER, L. AQUAD 6. Manual del programa para analizar datos cualitativos. 1. ed. 2003, Tübingen: Ingeborg Huber Verlag. Tübingen: Günter Huber, 2012.

IGLESIAS, M.; LOZANO, I.; MARTÍNEZ, M. La utilización de herramientas digitales en el desarrollo del aprendizaje colaborativo: análisis de una experiencia en Educación Superior. Revista Docencia Universitaria. REDU, Santiago de Compostela, v. 11, n.2, p. 333-351, 2013.

JOHNSON, D.; JOHNSON, R. T. An educational psychology success story: interdependence theory and cooperative learning. Educational Researcher, Washington, v. 38, n.5, p. 365-379, 2009.

MARINA, J. A. Los docentes, conciencia educativa de la sociedad. CEE Participación Educativa, Madrid, v. 16, p. 2-3, 2011.

MASON, J. Researching your own practice. The discipline of noticing. London: RoutledgeFalmer, 2002.

MCLAUGHLIN, M.; TALBERT, J. Building school-based teacher learning communities: professional strategies to improve student achievement. New York: Teachers College Press, 2006.

MILES, B.; HUBERMAN, A. Qualitative data analysis. London: SAGE, 1994.

MONTERO, L. El trabajo colaborativo del profesorado como oportunidad formativa. CEE Participación Educativa, Madrid, v. 16, p. 69-88, 2011.

NÓVOA, A. Para una formación de profesores construida dentro de la profesión. Revista de Educación, Madrid, v. 350, p. 203-218, 2009.

OPFER, V.; PEDDER, D. Conceptualizing teacher professional learning. Review of Educational Research, Washington, v. 81, n. 3, p. 376-407, 2011. 
PALOMARES, A. El modelo docente universitario y el uso de nuevas metodologías en la enseñanza, el aprendizaje y la evaluación. Revista de Educación, Madrid, v. 355, p. 591604, 2011.

PERRENOUD, P. Desarrollar la práctica reflexive en el oficio de enseñar: profesionalización y razón pedagógica. Barcelona: Graó, 2004.

STOLL, L.; BOLAM, R.; MCMAHON, A.; WALLACE, M.; THOMAS, S. Professional learning communities: a review of the literature. Journal of Educational Change, Netherlands, v. 7, p. 221-258, 2006.

VÁZQUEZ, E.; FOMBONA, J.; FERNÁNDEZ, A. Virtual attendance: analysis of an audio-visual over IP system for distance learning in the Spanish Open University (UNED). The International Review of Research in Open and Distance Learning, Canada, v, 14, n. 3, p. 402-426, 2013.

VESCIO, V.; ROSS, D.; ADAMS, A. A review of research on the impact of professional learning communities on teaching practice and student learning. Teaching and Teacher Education, Amsterdam, v. 24, n. 80-91, 2008.

WENGER, E. Communities of practice: learning, meaning and identity. Cambridge: Cambridge University Press, 1998.

WONG, S. Relationships matter: some benefits, challenges and tensions associated with forming a collaborative educational researcher group. Australian Educational Researcher, Netherlands, v. 41, n. 3, p. 243-259, 2014.

ZEICHNER, K. Rethinking the Connections Between Campus Courses and Field Experiences in College- and University-Based Teacher Education. Journal of Teacher Education, Washington, v. 61, n. 1-2, p. 89-99, 2010.

Recibido en 31 de Marzo de 2015.

Aprobado en 20 de Abril de 2016. 